\title{
Skin prick test for the evaluation of patients with idiopathic generalized pruritus: preliminary results
}

\section{Funda Tamer', Mehmet Eren Yuksel ${ }^{2}$}

\author{
${ }^{1}$ Ufuk University School of Medicine, Department of Dermatology, Ankara, Turkey \\ ${ }^{2}$ Aksaray University School of Medicine, Department of General Surgery, Aksaray, Turkey
}

Corresponding author: Dr. Funda Tamer, E-mail: fundatmr@yahoo.com

\begin{abstract}
Background: Pruritus is an unpleasant sensation which can decrease the patient's quality of life by leading to anxiety and sleep disturbances. Pruritus can present due to various dermatological and systemic diseases like eczema, xerosis, diabetes mellitus, cholestasis and uremia. However, no etiologic factor can be detected in some patients. Idiopathic generalized pruritus is used to describe pruritus without any underlying dermatosis or systemic disorders. Skin prick test is used for the evaluation of patients with generalized pruritus to determine the causative agent. Material and Methods: The skin prick test results of the patients with idiopathic generalized pruritus who were admitted to dermatology outpatient clinic between April 2017 and May 2018 were reviewed retrospectively. Results: This study included 18 patients (12 female, 6 male) with idiopathic generalized pruritus. The mean age of the patients was 38.5. The mean disease duration was 3.4 years. Eleven $(61.1 \%)$ patients had at least one positive reaction to skin prick testing. However, 7 (38.9\%) patients did not react to any allergens. The most common allergies were to trees mixture and Aspergillus mix. Conclusions: Skin prick testing can be helpful in the management of patients with idiopathic generalized pruritus. Hereby, the most common allergens were detected as trees mixture and Aspergillus mix. However, a larger sample size is needed to characterize the most common allergens in Turkish patients with idiopathic generalized pruritus.
\end{abstract}

Key words: Allergy; Generalized; Idiopathic; Itching; Pruritus; Skin prick test

\section{INTRODUCTION}

Pruritus is the unpleasant sensation that leads to scratching and irritation of the skin [1]. Pruritus may be generalized or localized [2]. It is one of the most common subjective symptoms in dermatology. Pruritus lasting more than six weeks is called chronic pruritus. The prevalence of chronic pruritus in the general adult population has been reported $13.5 \%$, recently [1]. It has been suggested that $60 \%$ of the elderly individuals aged 65 and over suffer from moderate or severe pruritus [1]. However, the prevalence of pruritus in elderly varies in studies. Goyal et. reported that pruritus was observed in only $5 \%$ of the patients aged 60 and above [3]. Pruritus can decrease the patient's quality of life by leading to anxiety, sleep disturbances, sexual dysfunction and attention problems [1].
Pruritus usually occurs as a result of dermatological conditions like xerosis and eczema [4]. However, various systemic disorders including diabetes mellitus, cholestasis, uremia, thyroid disorders, iron deficiency, polycythemia vera, essential thrombocytosis, malignancy, human immunodeficiency virus infection, hepatitis $\mathrm{C}$ infection and drug reactions may be the underlying cause of pruritus $[2,4]$. It has been suggested that a systemic etiology can be detected in $14 \%$ to $24 \%$ of patients complaining of generalized pruritus without any primary cutaneous cause [5].

Detailed medical history of patients with chronic pruritus is required to determine the etiology. Diagnostic assessment should include clinical characteristics of pruritus, presence of cutaneous lesions, previously diagnosed systemic and dermatological diseases, family

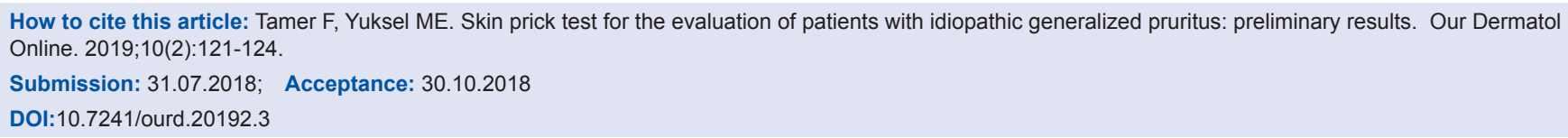


history, medications, allergies, and susceptibility to atopic dermatitis [6]. Evaluation of laboratory tests including complete blood count, liver function tests like alkaline phosphatase, alanine aminotransferase and aspartate aminotransferase, blood urea nitrogen, serum creatinine, ferritin and thyroid-stimulating hormone levels is recommended in patients without any dermatological and physical findings $[4,7]$. However, in $8 \%$ of all patients complaining of pruritus, no underlying dermatosis or systemic disorder can be detected. This condition is termed as idiopathic generalized pruritus $[7,8]$.

Hereby, we report the skin prick test results of the patients who had generalized pruritus without any underlying disorder or primary skin lesions.

\section{MATERIALS AND METHODS}

Medical reports of the patients with idiopathic generalized pruritus who were admitted to dermatology outpatient clinic between April 2017 and May 2018 were reviewed retrospectively. The exclusion criteria were pregnancy, malignancy, renal failure, use of systemic antihistamines, corticosteroids, tricyclic antidepressants, mast cell stabilizers and immunosuppressive drugs within four weeks.

The skin prick test was made on the flexural surfaces of the forearms (Figure 1). The test area was disinfected with $70 \%$ ethanol.

The skin prick test was performed using allergen extracts including Betula verrucosa, salicaceae, trees mixtures (locust, linden, horse chestnut

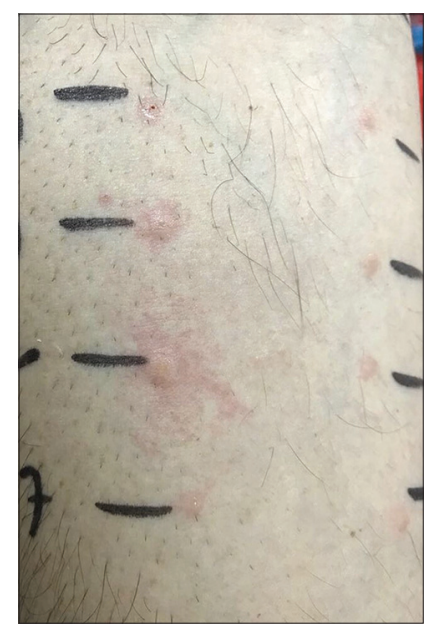

Figure 1: Erythematous papules on the flexor surface of the right forearm of the patient indicate a positive response. and plane tree), compositae, mixture of grasses, Dermatophagoides farinae, Dermatophagoides pteronyssinus, Cladosporium, Aspergillus mix, Felis domesticus (cat), dog hair, mixed feathers (duck, goose, hen), Poa pratensis, Pinus sylvestris, mixtures of cereals (barley, wheat, oats, corn), Secale cereale, latex, cockroach, mosquito, cocoa, olive, onion, paprika, pepper, tea, wheat flour, cereal grain mix, apple, banana, orange, peach, strawberry, peanut, hazelnut, walnut, tomato, egg (whole), chicken, positive control and negative control (Table 1).

Allergen solutions were placed on the flexural surfaces of both forearms at a two cm distance from each other. Small pricks were made into the skin through allergen extract drops using disposable plastic lancet. The excess solutions were removed gently using a paper towel to prevent the mixing of allergens with each other. The results were evaluated 15 minutes after the skin pricks. Allergen reactions were considered positive when the wheal diameter was $3 \mathrm{~mm}$ or larger than the negative control (Figure 1).

\section{RESULTS}

The study included 18 patients (12 female, 6 male) with idiopathic generalized pruritus. The mean age of the patients was 38.5 (range: 18-47). The mean disease duration was 3.4 years (range: 2 months- 15 years).

The past medical history was remarkable for angioedema in one patient, rosacea in one patient, allergic rhinitis in two patients and hyperlipidemia in two patients. Seven

Table 1: Allergen extracts used for the skin prick test

\begin{tabular}{ll}
\hline Allergens used for skin prick test & \\
\hline 1 Positive control & 21 Mosquito \\
2 Negative control & 22 Cocoa \\
3 Betula verrucosa & 23 Olive \\
4 Salicaceae & 24 Onion \\
5 Trees mixture & 25 Paprika \\
6 Compositae & 26 Pepper \\
7 Mixture of grasses & 27 Tea \\
8 Dermatophagoides farinae & 28 Wheat flour \\
9 Dermatophagoides pteronyssinus & 29 Cereal grain mix \\
10 Cladosporium & 30 Apple \\
11 Aspergillus mix & 31 Banana \\
12 Felis domesticus (cat) & 32 Orange \\
13 Dog hair & 33 Peach \\
14 Mixed feathers & 34 Strawberry \\
15 Poa pratensis & 35 Peanut \\
16 Pinus sylvestris & 36 Hazelnut \\
17 Mixtures of cereals & 37 Walnut \\
18 Secale cereale & 38 Tomato \\
19 Latex & 39 Egg \\
20 Cockroach & 40 Chicken \\
\hline
\end{tabular}


patients did not receive any prior treatment. However, 11 patients were treated with topical corticosteroids, emollients, oral antihistamines or intramuscular injections of corticosteroids previously.

The skin prick test results of the patients are depicted in Table 2. Eleven $(61.1 \%)$ patients had at least one positive reaction to skin prick testing. However, $7(38.9 \%)$ patients did not react to any allergens (Table 2).

\section{DISCUSSION}

Skin prick test is a quick, safe and minimally invasive method to detect reactions to food, drug, inhalant and occupational allergens [9]. Skin prick test is usually performed to investigate the allergens in patients with rhinoconjunctivitis, contact urticaria, asthma, atopic eczema, and suspected food allergy [10]. As the reaction to a specific allergen is localized, various allergens can be used at the same time. The prick test can be repeated to identify new sensitization in case of exposure to a new suspicious environmental allergen. Moreover, epidemiologic studies which determine sensitization rates in different areas contribute to create standardized allergen solutions [9]. Most common aeroallergens are house dust mites, pet dander, pollens and moulds, whereas commonly detected food allergens in adults include shellfish, nut and fruit allergies [11].

The skin prick test results of the patients with idiopathic generalized pruritus have been reported in a few study. Colgecen et al. reviewed skin prick test results

\begin{tabular}{ll}
\multicolumn{2}{l}{ Table 2: The skin prick test results of the patients } \\
\hline Patients & Allergens with positive skin reaction \\
\hline 1 & Hazelnut \\
2 & - \\
3 & - \\
4 & - \\
5 & - \\
6 & Aspergillus mix, mosquito, chicken \\
7 & Mixture of grasses, poa pratensis, mixtures of cereals, secale \\
8 & cereale, banana \\
9 & - \\
10 & Trees mixture, compositae, felis domesticus, cocoa, olive, \\
11 & paprika, tea \\
12 & Aspergillus mix, mosquito, banana \\
13 & Mixture of grasses, strawberry, egg \\
14 & Peanut, hazelnut \\
15 & Trees mixture, poa pratensi \\
16 & - \\
17 & Trees mixture, aspergillus mix \\
18 & Tomato \\
\hline
\end{tabular}

of 190 patients with idiopathic generalized pruritus, atopic dermatitis, chronic idiopathic urticaria, alergic rhinitis and asthma. The study revealed that 28 of the 53 patients with idiopathic generalized pruritus (52.8\%) had at least one or more positive reaction to the skin prick test. Banana, Dermatophagoides pteronyssinus and latex were the most common allergies in the group of idiopathic generalized pruritus. However, pine pollen, wheat pollen and dog epithelium were the most common allergies in all of the patients [12].

Furthermore, Baz et al. investigated the skin prick test results of 127 patients with idiopathic generalized pruritus, atopic dermatitis and chronic idiopathic urticaria. Baz et al. revealed that 23 of the 52 patients with idiopathic generalized pruritus (44.2\%) had at least one or more positive reaction to the skin prick test. Dermatophagoides pteronyssinus was the most common allergen in the group of idiopathic generalized pruritus. However, Dermatophagoides farinae and Dermatophagoides pteronyssinus were the most common allergens in all patients, respectively [13].

Stockli et al. reported a 26-year-old male patient with a 2-month history of generalized itching without any specific skin lesion. The past medical history was remarkable for non-seasonal rhinoconjunctivitis and consumption of cannabis and tobacco. Prick test showed positive reaction to the patient's cigarette and cannabis. Following the avoidance of only cannabis the symptoms regressed partially, without any treatment [14].

Vakaljan et al. presented a patient with chronic pruritus following the initiation of vitamin B12 supplementation for the treatment of pernicious anemia. Skin prick testing to nickel, cobalt chloride, and palladium chloride were identified as positive. Vakaljan et al. reported that the patient healed three days after the cessation of oral vitamin B12 supplementation. Sensitization to cobalt was reported to be a triggering factor for chronic pruritus [15].

In our study, skin prick test results of 18 patients with idiopathic generalized pruritus were reviewed. Most of the patients $(61.1 \%)$ showed positive reaction to various allergen extracts. The most common allergies in Turkish patients were to trees mixture and Aspergillus mix. The test result of a patient showed a positive reaction even to tea, which is frequently consumed in Turkey. Hereby, the preliminary results of skin prick test for the evaluation of patients with generalized pruritus have been reported. As our data increase, we would like to 
determine and share the most common allergens in Turkish patients with idiopathic generalized pruritus with our colleagues.

\section{Statement of Human and Animal Rights}

All procedures followed were in accordance with the ethical standards of the responsible committee on human experimentation (institutional and national) and with the Helsinki Declaration of 1975, as revised in 2008.

\section{Statement of Informed Consent}

Informed consent was obtained from all patients for being included in the study.

\section{REFERENCES}

1. Rajagopalan M, Saraswat A, Godse K, Shankar DS, Kandhari S, Shenoi SD, et al. Diagnosis and management of chronic pruritus: an expert consensus review. Indian J Dermatol. 2017;62:7-17.

2. Feramisco JD, Berger TG, Steinhoff M. Innovative management of pruritus. Dermatol Clin. 2010;28:467-78.

3. Goyal A, Balai M, Mittal A, Khare AK, Gupta LK. Pattern of geriatric dermatoses at a Tertiary Care Teaching Hospital of South Rajasthan, India. Our Dermatol Online. 2017;8:237-41.

4. Nowak D, Yeung J. Diagnosis and treatment of pruritus. Can Fam Physician. 2017;63:918-24.

5. Reamy BV, Bunt CW, Fletcher S. A diagnostic approach to pruritus.
Am Fam Physician. 2011;84:195-202.

6. Grundmann S, Ständer S. Chronic pruritus: clinics and treatment. Ann Dermatol. 2011;23:1-11.

7. Millington GWM, Collins A, Lovell CR, Leslie TA, Yong ASW, Morgan JD, et al. British Association of Dermatologists' guidelines for the investigation and management of generalized pruritus in adults without an underlying dermatosis, 2018. Br J Dermatol. 2018;178:34-60.

8. Yosipovitch G, David M. The diagnostic and therapeutic approach to idiopathic generalized pruritus. Int J Dermatol. 1999;38:881-7.

9. Heinzerling L, Mari A, Bergmann KC, Bresciani M, Burbach G, Darsow U, et al. The skin prick test - European standards. Clin Transl Allergy. 2013;3:3.

10. Berger A. Skin prick testing. BMJ. 2002;325:414.

11. Coetzee O, Green RJ, Masekela R. A guide to performing skin-prick testing in practice: tips and tricks of the trade. S Afr Fam Pract. 2013;55:415-9.

12. Colgecen E, Ozyurt K, Intepe YS, Kapusuz Gencer Z, Ozkiris M, Ede $G$, et al. Skin prick test results in patients with atopic symptoms in Yozgat district. J Clin Exp Invest. 2014;5:64-8.

13. Baz K, Guvenc U, Cordan Yazici A, Kokturk A, Ikizoglu G, Tasdelen B. Atopy and skin diseases in Mersin. Turkiye Klinikleri J Dermatol. 2007;17:105-11.

14. Stockli SS, Bircher AJ. Generalized pruritus in a patient sensitized to tobacco and cannabis. J Dtsch Dermatol Ges. 2007;5:303-4.

15. Vakaljan S, Ohayon Hamilton J. Cobalt as the culprit: vitamin B12 supplementation as a trigger for chronic pruritus. Ann Allergy Asthma Immunol. 2017;119:S17-96.

Copyright by Funda Tamer, et al. This is an open-access article distributed under the terms of the Creative Commons Attribution License, which permits unrestricted use, distribution, and reproduction in any medium, provided the original author and source are credited.

Source of Support: Nil, Conflict of Interest: None declared. 